\title{
Developing Mathematical Models and Methodologies to Overcome Lean Strategy Selection and Leanness Assessment Problems
}

\author{
Rakhi Ashok Deshpande, P. Venkata Chalapathi
}

\begin{abstract}
This investigation inspects the impact of lean strategies among chose manufacturing organizations. In orderto remain focused; creating cheaper products at a quicker rate Lean Manufacturing would encourage the business. In this paper we discuss about adopting lean strategies by manufacturing industries with the intention of eliminating wastes. Here we create mathematical models and a systematic methodology for choosing fitting lean strategies. The main objectives of this paper are to assess the leanness levels in manufacturing organizations. In the work we will evaluate both quantitative and subjective info factors. Different tools like MATLAB, Microsoft Access and Excel, Visual Basic, DST tool will be used for developing mathematical models and methodologies to overcome lean strategy selection and leanness assessment problems. As a result we try to limit the conceivable disarray of executing different lean strategies which can be utilized by any manufacturer for surveying leanness and distinguishing their leanness benchmark Accordingly, we suggest that manufacturing firms nearby different types of organizations (benefit firms comprehensive) ought to receive lean reasoning, introduction or culture to dispense with wasteful practices in this way clearing a path for best practices that can attract main concern for the organizations.
\end{abstract}

Index Terms: Lean, strategies, manufacturing, organization, industries, models, etc.

\section{INTRODUCTION}

Manufacturing tasks are ceaselessly endeavoring to expand profitability and yield of their activities. They will probably fulfill the client with the correct item, quality, amount, and cost in the briefest measure of time. Lean manufacturing is in excess of a cost decrease program or a critical thinking approach. The principle thought is that a proficient creation can be accomplished by a far reaching way to deal with limit wastes. This implies taking out overabundance creation and stock, repetitive development of material, pausing and delays, over handling, abundance laborer movement, and the requirement for modify and

Revised Manuscript Received on October 30, 2019.

* Correspondence Author

RAKHI ASHOK DESHPANDE, Research Scholar, Department of Mechanical Engineering, KoneruLakshmaiah Education Foundation, Vaddeswaram, Andhra Pradesh, 522502, India. Aadhaar Number - 575756459875.

DR. P. VENKATA CHALAPATHI , Professor, Department of Mechanical Engineering, KoneruLakshmaiah Education Foundation, Vaddeswaram, Andhra Pradesh, 522502, India.

(C) The Authors. Published by Blue Eyes Intelligence Engineering and Sciences Publication (BEIESP). This is an open access article under the CC BY-NC-ND license (http://creativecommons.org/licenses/by-nc-nd/4.0/) remedies. Some portion of lean manufacturing is checking on activities for those parts, procedures or items that include cost as opposed to esteem. Each progression of the manufacturing procedure is observed to decide whether it increases the value ofthe item. In the event that it doesn't include Value, the procedure could be appointed to a subcontractor or redistributing organization with the end goal to concentrate the staff on esteem included tasks of its center business. Endless number of manufacturing organizations has made upgrades since the presentation of Lean management strategies and apparatuses. In any case, the advantages have not been as so great for service industries applying Lean management standards.

A. Concept of lean manufacturing and lean manufacturing strategies

Lean Manufacturing can be characterized as: Lean manufacturing or lean generation, which is regularly known just as" Lean", is the ideal method for producing goods through the evacuation of waste or we can say "Lean manufacturing is the system which points in end of the loss from the system with a systematic and constant methodology" OR Lean Manufacturing is an operational system arranged toward accomplishing the briefest conceivable process duration by disposing of waste. Lean manufacturing techniques depend on the utilization of five principles to manage management's activity toward progress.

A lean organization fathoms customer esteem and centers its key processes to constantly expand it. This strategy thinks about the regard for the laborers, the nature of the products and the steadiness of the process. This production method changes the focal point of management and guides it to the disposal of waste along whole value streams, rather than at individual focuses. To achieve this makes processes that need less human exertion, less space, less capital, and less time to make products and services at far less costs and with many less imperfections, contrasted and customary production systems. There are five fundamental ideas that characterize lean reasoning and empower lean generation: determine esteem; distinguish the esteem stream, stream, force, and error free. Clearly, lean reasoning underlines quality and incentive for every item from the point of view of the end client. Lean manufacturing, spearheaded by Toyota, includes stock and quality control, modern relations, work administration, and provider producer rehearses that vary on a very basic level from customary business rehearses. This attestation takes us to the five principle of lean thinking: Value, Value Stream, Flow, Pull and perfection. 


\section{Developing Mathematical Models and Methodologies to Overcome Lean Strategy Selection and Leanness Assessment Problems}

- Include Value: Value is characterized as a "capacity given to customer at the correct time and at a correct cost, as built up for each situation by the customer".

- Value Stream: The value stream is characterized in Lean Thinking as the arrangement of all the "particular exercises required to configure, arrange, and give a particular item, from idea to dispatch, request to conveyance, and crude materials under the control of the customer"

- Production Flow: Flow is characterized as the "dynamic accomplishment of errands along the value stream so an item continues from configuration to dispatch, request to conveyance and crude materials under the control of the customer without any stoppages, scrap or reverses".

- Pull System: The fourth lean principle of pull is characterized as a "system of falling production and conveyance guidelines from downstream to upstream in which nothing is created by the upstream provider until the point that the downstream customer flags a need"

- Perfection: The fifth and last lean principle is perfection, characterized as the "total disposal of waste with the goal that all exercises along a value stream make value".

\section{B. Role of lean manufacturing in waste management}

Lean is typically accomplished with the utilization of tools, including botch demonstrating, value stream mapping, visual management and pull-production, which are world-class tools and techniques successfully connected in different territories, for example, the vehicle segment. This rationality of enhancement through waste decrease is alluded to as lean methodology. Lamentably, notwithstanding the wide multifaceted nature of operations engaged with the machine tool manufacturing industry, complete test examinations on machine tool manufacturing utilizing lean methodologies are as yet insufficient. In the machine tool manufacturing industry, directed wastes for decrease or disposal incorporate blemished processed parts, pointless transportation of laborer and materials at the shop floor, extreme in-process inventories, sitting tight for guidance from the better on the following move than make amid operations processing, save parts over-production and over processing of parts and segments. These wastes must be decreased or dispensed with for better intensity of the business. Positively, more research is fundamental in the lean manufacturing region as exemplified by the economic emergency around the world, which has brought about violent environmental conditions for machine tool manufacturing practices

\section{Relation between lean manufacturing and business success}

Lean production is the most mainstream investment decision among organizations. The process contains pull production, quality advancement, process center, consistent enhancement, value stream management, and laborer strengthening. The goal of LP is to fulfill customer demands on the most astounding conceivable level through waste decrease. End of wastes can be considered in the HR, plan, production processes and exercises, circulation, and inventory segments. In the event that few sorts of wastes can be wiped out, how do organizations rehearse the key principles of lean to fabricate versatile, flexible, and inventive supply chains? What are the proper tools and techniques of the lean supply chain? Supply chain experts are chipping away at intends to diminish wasted exertion and non-value exercises over the supply chain. As indicated by the idea of LP, actualizing its tools and techniques help limit such wasted exertion. Amid the initial steps, supply chain accomplices ought to understand the lean idea, and then actualize its practices through abnormal amounts of coordinated effort and cooperation. Most articles on LP center around the usage of systems, for example, without a moment to spare (JIT), add up to quality management (TQM), add up to preventive upkeep programs, human asset management, value stream mapping, and merchant improvement, and additionally their effect on operational performance. Fewer examinations research the usage of the lean idea in the supply chain, and distinguish the most imperative tools and techniques that complete the goals of the lean idea in the supply chain.

\section{REVIEW OF LITERATURE}

Pentlicki, J.H. (2014) Based on who built up a more profound comprehension of the boundaries looked by SMEs and the methodologies required for the effective implementation of lean manufacturing, senior pioneers have fluctuating definitions of their jobs in driving lean manufacturing implementation, have varying points of view with respect to the level of authority information required for fruitful lean manufacturing implementation. They likewise battle to grow lean manufacturing implementation into support divisions, for example, building, acquiring, regulatory functions, and deals. This means all representatives need to find out about lean, paying little respect to where in the organization they work. Be that as it may, the learning procedure should be different for the board than for every single other worker, which is considered in our new model.

Bortolotti, T., Boscari, S., Danese, P. (2015) In the examination hierarchical culture and lean practices were explored in connection to effective and unsuccessful lean implementation. The creators reasoned that a fruitful lean plant has a higher institutional collectivism, future orientation, human orientation, utilization of little gathering problem-unraveling, advancement of provider partnership, customer involvement, reception of consistent enhancement, and lower assertiveness in correlation with unsuccessful lean plant. The results likewise show, that so as to actualize lean administration effectively it is principal to go past lean administration details by receiving delicate practices and supporting the improvement of a fitting authoritative culture profile.

Kull, T.J., Yan, T., Liu, Z., Wacker, J.G. (2016) expressed that the effective utilization of lean manufacturing rehearses requires more than the utilization of instruments. It relies upon a country's culture, and in addition the organization culture. The culture in an organization relies upon an example of the administration, and a culture change is a long procedure which can be made by LP with the board support.

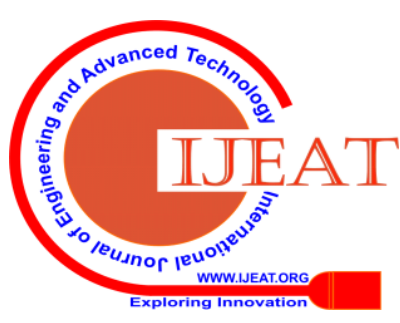


Jekiel, C.M. (2017) gives directors and officials the means how to boost worker potential by expanding the enhancement control. It additionally characterizes the general population related methodologies and practices expected to modify any social dynamic. The creators expressed, that everybody needs to learn and enhance, and must be included. They propose a five-year intend to make a durable change requiring development of association, culture, and conduct

\section{OBJECTIVES OF THE STUDY}

1. To create mathematical models and a systematic methodology for choosing fitting lean strategies

2. To assess the leanness levels in manufacturing organizations.

\section{METHODOLOGY}

A leanness appraisal model will be created by using the fluffy idea to survey the leanness level and to prescribe an ideal leanness value for a manufacturing organization. In the proposed leanness evaluation show, both quantitative and subjective info factors are to be considered. In view of program created in MATLAB and C\#, a decision support tool (DST) will be produced for decision creators to choose lean strategies and assess the leanness value dependent on the proposed models and methodology thus maintain the lean execution efforts. A contextual investigation will be directed to show the adequacy of these models and methodology.

\section{A. Parameters Used}

The data for the exploration will be gathered through different sources, they are: writing studies officially done in the field, firm investigations, Meetings, observations, Value stream mappings, interviews, time and motion analysis, quantitative and qualitative data analysis.

\section{B. Tools}

Different tools that will be utilized to finish the exploration work are MATLAB, Microsoft Access and Excel, Visual Basic, DST tool.

\section{RESULT \& ANALYSIS}

A lean strategy selection module has been created to illuminate the conditions and to accomplish ideal arrangement. Lean strategy selection module is produced dependent on MATLAB and Excel spreadsheet. This module helps to find appropriate lean strategies for identified wastes within manufacturer resource constraints applying developed mathematical models and proposed methodology.

\section{A. Mathematical Model for estimating perceived value, Lean Implementing Cost and Time}

Seen value means the manufacturers' impression of diminishing a manufacturing waste. In this exploration, maker discernment is measured by giving a relative significance value to their objective.

The expansion of significance of decrease of any loss for a producer is considered as the expansion in saw value file. This examination additionally creates cost and time list to evaluate the required cost and time to execute a lean strategy. In this model, the cost indexes of lean implementation are incorporated into the form of working cost, speculation cost, and variable cost. Time list of lean implementation is incorporated into the form of planning time for lean implementation, alteration time of the leaving process, training time required for the operators and management personnel about lean systems, and approval time for the new or lean creation process

\section{i. Value index of lean implementation}

The goal of executing a lean strategy in a manufacturing process is to decrease waste and increment productivity with least cost and time. At the point when a change in a framework does not add to one of the goals, at that point it is considered a nonvalue included endeavor. Therefore, these changes ought not to be sought after further. This exploration considers the accompanying two sub-goals to achieve the above destinations;

- Maximize the apparent value of lessening a manufacturing waste by actualizing lean procedures.

- Minimize the implementation cost and time

(In references Gautam and singh not found) As indicated by Gautam and Singh, an expansion in the perceived value index by executing $\mathrm{n}$ lean procedures can be communicated as;

(1)

$$
\sum_{1}^{n} L_{i} S_{1}
$$

On the off chance that two strategies have some between reliance and every strategy has an impact on others, at that point the additional change in perceived contribution value can be communicated as;

$$
\sum_{1}^{n} \sum_{1}^{n} L_{i} L_{j} \delta_{2 i f}
$$

In Equations (1) and (2), if a lean strategy is chosen for implementation and is 1 (i.e. $L_{\mathrm{i}}=1$ ) and 0 (i.e. $=0{ }^{L_{\mathrm{i}}}$ ) on the off chance that it isn't chosen for implementation. Therefore, implementation of the ith lean strategy contributes $1 \mathrm{i} \delta$ towards a manufacturer perceived value. Also, on the off chance that ith and jthstrategies are coupled so that implementation of strategy forces a change in $\mathrm{j}$ th strategy, at that point the subsequent contribution towards the manufacturing perceived value due to $L_{\mathrm{i}}$ is $\delta_{2 i \mathrm{ij}}$. Therefore, add up to change in the perceived value index is the summation of perceived value without executing a lean strategy, perceived value due to actualizing a lean strategy and perceived value of forced changes because of lean implementation

\section{ii. Cost and time index for lean implementation}

In this model, the costs of lean implementation are incorporated into the form of working cost, venture cost, and variable cost. Time of lean implementation is incorporated into the form of planning time for lean implementation, change time of the leaving process, training time required for the refreshed framework, and approval time for the new creation process. 


\section{Developing Mathematical Models and Methodologies to Overcome Lean Strategy Selection and Leanness Assessment Problems}

\section{Operating cost index}

In the event that $L_{\mathrm{i}}$ is the portrayal of $\mathrm{i}^{\text {th }}$ lean strategy for decreasing a specific manufacturing waste and $C_{P_{U}}$ is the required working cost of this lean tool implementation; at that point the working cost of lean strategy implementation in the current framework can be communicated as:

$$
\sum_{1}^{n} L_{1} C_{1 i}
$$

\section{Investment cost index}

Interest in cost of change relies upon the intricacy of the adjustment required in the current manufacturing process to actualize a lean strategy and the dimension of lean strategy implementation. In the event that $L_{\mathrm{i}}$ is the portrayal of $\mathrm{i}^{\text {th }}$ lean strategy for lessening a manufacturing waste and $C_{\mathbb{l}_{v}}$ is the venture cost required for actualizing the $\mathrm{i}^{\text {th }}$ strategy; at that point the speculation cost of the expected change of the current framework can be communicated as;

$$
\sum_{1}^{n} L_{i} C_{1_{1 i}}
$$

\section{Variable cost index}

Variable costs are those costs which fluctuate relatively with the volume of units created. Variable costs are an immediate function of production volume, rising at whatever point production extends and falls amid its agreements. Examples of common variable costs include: crude materials, bundling, and work straightforwardly engaged with an organization's manufacturing process. When $I V_{v 1}$ is variable cost, $v(i)$ is production volume, and $L_{\mathrm{i}}$ is the portrayal of $\mathrm{i}^{\text {th }}$ lean strategy, at that point change in factor cost index can be determined as;

$$
\sum_{1}^{n} v(i) L_{i} V_{v_{11}}
$$

\section{Planning time index}

Implementation of lean procedures or strategies in the current framework requires planning from the best management before implementation. A few planning exercises are required to put by and by a new improvement strategy, for example, planning for improvement of functional necessities, offices advancement, planning for implementation process and strategies.

On the off chance that $L_{\mathrm{i}}$ is the portrayal of $\mathrm{i}^{\text {th }}$ lean strategy for diminishing a specific manufacturing waste and $T_{P 1 t}$ is the required planning time for this lean strategy implementation; at that point the aggregate planning time required for executing $n$ strategies and overhauling the current manufacturing framework;

$$
\sum_{1}^{n} L_{i} T_{P_{1 i}}
$$

\section{iii. Decision Function}

The goal of this examination is to expand the manufacturer perceived value of decreasing the distinguished manufacturing wastes by executing proper lean strategies inside their restricted cost and time. Scientifically, it tends to be communicated as;

Perceived value from lean implementation $=$

$$
\delta_{o_{i}}=\sum_{1}^{n} L_{i} \delta_{1_{i}}+\sum_{1}^{n} \sum_{1}^{n} L_{i} L_{f} \delta_{2 i f}
$$

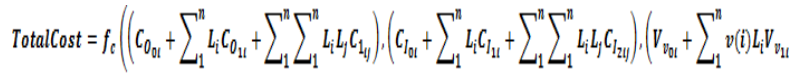

$$
\begin{aligned}
& \left.\left.+\sum_{1}^{n} \sum_{1}^{n} v(j) L_{i} V_{v_{2 l}}\right)\right)
\end{aligned}
$$

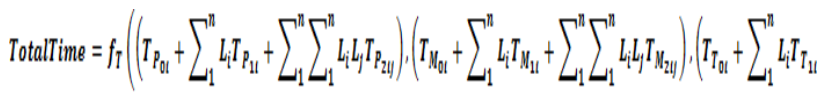

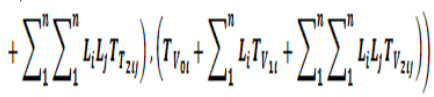

\section{iv. Constraints}

No manufacturing association has boundless assets and spending plan to actualize a new strategy.

Therefore, any new advancement program has targets and asset requirements and the real cost and time based limitations are considered in this examination:

Operating cost:

$$
C_{o_{0 u}}+\sum_{1}^{n} L_{i} C_{o_{1 i}}+\sum_{1}^{n} \sum_{1}^{n} L_{i} L_{j} C_{o_{z y}} \leq O C_{C}
$$

Investment cost:

$$
C_{I_{0 i}}+\sum_{1}^{n} L_{i} C_{I_{14}}+\sum_{1}^{n} \sum_{1}^{n} L_{i} L_{j} C_{I_{z l y}} \leq I C_{C}
$$

\section{B. Description of Detailed Model using Case Study}

The company (ABC Power Switchgear Pty) specializes in the research and development, make, marketing, deals and administration of low, medium and high voltage switchgear products. ABC Power has three fundamental departments: Research and Product development, Supply Chain Management (SCM) and Production. Research and Product development department plans the product dependent on client details and market demand. The SCM department handles the associations with suppliers and acquires the crude materials. It is likewise in charge of the arranging and control of the production processes. The Production department creates the products, performs product testing and quality affirmation. ABC needs to meet the stringent necessities of the mining and process control industries. Today the company is spoken to in excess of 50 nations and their products and administrations have a notoriety of high dependability, driving edge innovation and long life. So as to remain aggressive, the company is quick to grasp lean manufacturing strategy to enhance its productivity and quality by conquering the conceivable wasteful aspects in the production processes. Before, ABC executed some enhancement strategies in their production process (OSM300 assembly line, for example, Kanban bin and Rapid Performance Management (RPM) so as to enhance the production productivity. 
In any case, ABC neglected to accomplish huge advantages from that usage because of their wrong observation about lean. They imagined that executing lean strategies will dependably limit the measure of all assets utilized in the different exercises in the production processes. Be that as it may, they didn't know about related expense and time required for lean execution.

i) Project team formation: A lean team was shaped in this project containing diverse management and production work force. The team individuals included: the engineering manager, production manager, one mechanical engineer, one researcher (Author), and one skilled operator.

ii) Determination of the investigation: The principal undertaking of the lean team was to decide the examination scope. Subsequent to examining with the ABC's management, the lean team concluded that they will use the created mathematical models and technique for choosing fitting lean strategies for the OSM300 assembly line, as ABC neglected to accomplish huge advantages in this assembly line in their prior endeavor. OSM300 assembly line is a devoted assembly line for delivering automatic circuit recloser named OSM300-38kV reclosers. The OSM300-38kV automatic circuit reclosers is intended for utilizing on overhead appropriation lines and in addition circulation substation applications for voltage classes up to $38 \mathrm{kV}$. To examine the OSM300 assembly line, lean team drew an esteem stream mapping of current state guide of the OSM300-38kV assembly line. The primary motivation behind the current state delineate esteem stream mapping was to distinguish the non-esteem including ventures in OSM300-38kV assembly process. Figure 1 introduces the current state guide of the OSM300 assembly process.

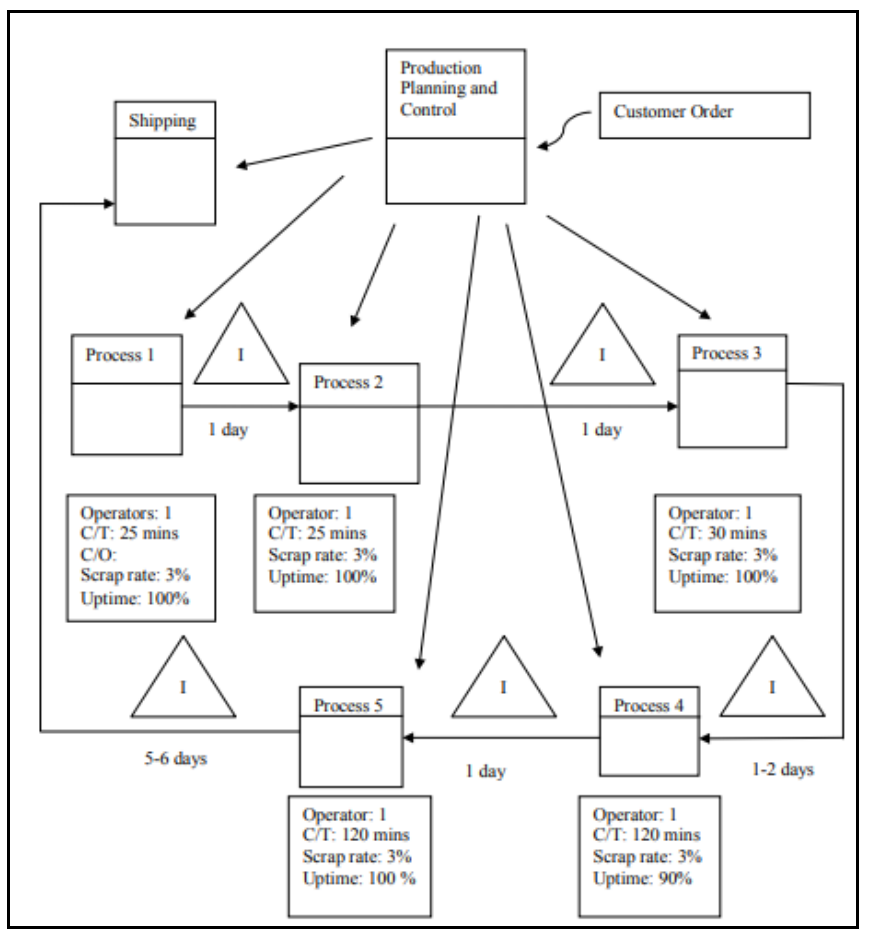

Fig. 1: Current State Map of OSM300 Assembly Process

We mean the stock of the process, $\mathrm{C} / \mathrm{T}$ is the process duration of the specific process, $\mathrm{C} / \mathrm{O}$ is the changeover time, and Up time was determined by subtracting downtime because of disappointments. In the current state of the OSM300 assembly line, raw material remains in the store for three to four days. Then, in light of the week after week schedule, raw materials are withdrawn from the store to make OSM300-38kV reclosers. The OSM300-38kV collecting begins from the installation of VI boot in the shafts at Workstation 01 (W01). Then, the armature is embedded into the VI boot in the Workstation 02 (W02). Next, the continuance test is completed in Workstation 03 (W03), and then Gang in bar is introduced in the Workstation 04 (W04) and at long last, posts are introduced in the tank in Workstation 05 (W05). Subsequent to playing out a few movement and quality tests, at long last, products are transported to the customer. Notwithstanding, it was further chosen by lean team and ABC's best management to concentrate just on W04 (i.e. group in bar installation arrange) because of project finishing time limitations and ABC's classification issues. ABC's management additionally concurred that they encountered W04 as their significant hindrance of OSM300 line to take care of the customer demand due its low effectiveness. They additionally stated that W04 takes additional time than other workstations to finish a product which makes late conveyance the customer arrange. In addition, it was chosen that in the event that time licenses, lean team can expand their work on the other workstations also. Because of these above restrictions, this investigation just spotlights on W04. We will talk about the relationship among the distinctive workstation of OSM300 assembly line. The assembly line is then improved concentrating on W04 to understand the principle exercises of W04 and modified assembly line is given. W04 is considered as an entire manufacturing system, W03 and distribution center is its provider, and W05 is its customer.

iii) Identify the manufacturing wastes: Value stream mapping (VSM) recognizes the presence of wastes in the distinctive phases of OSM300 assembly line. Amid watching the process, it was recognized that there are a few wastes that esteem stream mapping can't discover which can be distinguished utilizing other methods. Instances of these wastes include: unneeded movement, pausing, sharing tools, and transportation of parts and tools. Therefore, video recording of the process and then time think about examination, observations, unstructured meeting were viewed as extra complimentary methods with the esteem stream mapping to identify the other wastes for this case. Therefore, informal interviews, personal observations, and archival documents, video recording were utilized to identify the fundamental sources of wastes in W04

a) Time and motion study of Workstation 04-Time study is a Warehouse Supplier (W03) Customer (W05) Workstation 04 specific strategy that encourages manager to understand operator's whole working process, so as to highlight issues and infer proper arrangements.

\section{Published By:}

Blue Eyes Intelligence Engineering \& Sciences Publication

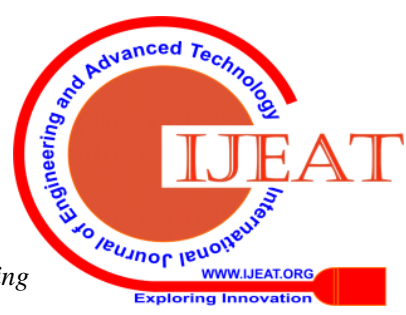


b) Process recording: Before process recording, lean team talked about the reason for video recording and recording method to the operators. . Video record and time study information for OSM300-38 kV at W04 are accessible on demand

c) Break down of recorded time-The lean team checked on the recorded video and broken it into time fragments that speak to every one of the subtleties of work process.

d) Categorize the process-After the time recording and break down into time portion, the project team examined the work process with the engineering manager and significant operator to decide if the process was of esteem added or nonvalue added category. Esteem added category is the action that must need to finish the product. Non-esteem added category is the action that does not increase the value of the last product or can be dodged to finish the product.

The identified nine wastes categorises are provided below:

- Walk to parts

- Walk to/get tools

- Handling/waiting

- Adjust/inspection

- Get parts

- Paperwork

- Unpacking/disassembly

- Cleaning

- Rework

e) Sketch non-value added and value added time spread-subsequent to assessing the time fragments an exceed expectations spread sheet is utilized to create a bar chart to identify the aggregate processing time.

f) Time study result analysis - The handy cam records the time to finish the whole assembly process at W04. It demonstrates the time required by a skilled operator to finish an occupation at W04 with no unsettling influence from internal or outer components. Components could be a section and apparatus deficiency in the workstation, and late occupation conveyance from W03 or distribution center etcetera. This was really a best case circumstance. Then, the whole process was separated into 796 motions seeing the video record. The whole process took about 121.79 minutes to finish this assembly arrange. The team additionally discovered that, the operator spent the greater part of the time or about 66.70 minutes on non-value adding tasks and just 55.16 minutes on value-adding tasks. The team then recognized the measure of non-value added time in the distinguished nine categorizes. The extents of non-value-added times of existing assembly process in W04 are exhibited in Table 1.

Table 1: Proportions of Non-value-added Times for existing Assembly Process in W04

\begin{tabular}{|l|l|l|}
\hline Activities name & $\begin{array}{l}\text { Time } \\
\text { spent }\end{array}$ & $\begin{array}{l}\text { Time } \\
\text { In Percent }\end{array}$ \\
\hline Value-added Time (VAT) & 55.16 & $45.5 \%$ \\
\hline
\end{tabular}

\begin{tabular}{|l|l|l|}
\hline Walk to parts & 4.50 & $3.4 \%$ \\
\hline Walk to/get tools & 9.05 & $7.3 \%$ \\
\hline Handling/waiting & 15.38 & $12.3 \%$ \\
\hline Adjust/inspection & 16.28 & $13.7 \%$ \\
\hline Get parts & 12.22 & $10.0 \%$ \\
\hline Paperwork & 0.00 & $0.0 \%$ \\
\hline Unpacking/disassembly & 1.93 & $1.8 \%$ \\
\hline Cleaning & 0.75 & $0.7 \%$ \\
\hline Rework & 6.52 & $5.3 \%$ \\
\hline Total & 121.79 & $100.0 \%$ \\
\hline
\end{tabular}

These non-value added times were further examined and it was discovered that larger part of the wastes (around 43\%) originated from strolling, holding and handling the parts and tools, investigation and getting parts to the assembly point. Figure 2 demonstrates the non-value-added times (red bar) and value-added time (green bar) related with the assembly exercises of W04.

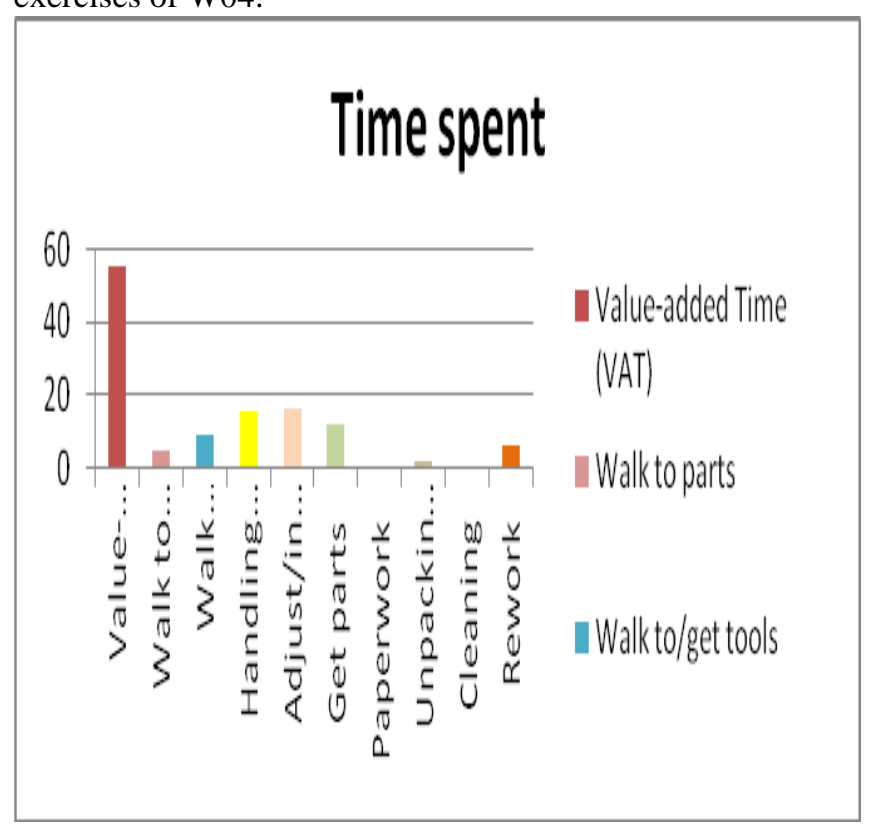

Fig. 2: Time Study Result Analysis

iv) Assignment of perceived values to reduction of wastes: After identifying the distinctive wastes, a perception and unstructured meeting process was likewise led on the factory floor to investigate further wastes related with the W04. This served to identify the current manufacturing problems which are categorized into ten sorts and then, evaluated by both the lean team and company officials. The lean team specifically asked the ABC's management individuals like engineering director and engineering manager their discernment about these wastes.

v) Establish relationship between W04's wastes and lean strategies and allocate perceived value to reduction of each waste: Planning to decrease manufacturing wastes includes implementation of at least one lean manufacturing strategies with the target that each lean manufacturing strategies that is actualized will add to diminish one of the recognized wastes. This progression finishes the lean strategies and wastes correlation matrix created subsequent to putting the relative significance values.

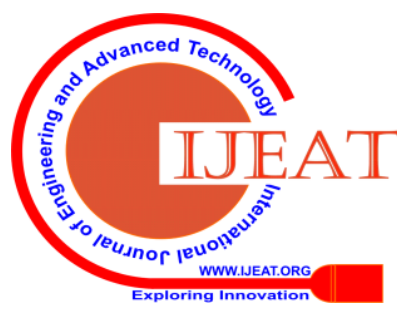


vi) Lean implementation cost and time: Resources are important to actualize any new tools or strategies in a current system. Therefore, the exertion required to make the change to lean manufacturing ought not be disparaged. Because of the absence of implementation cost and time data, this research assessed these costs and time data. This area assessed the measure of cost and time required for every strategy in cost and time units dependent on the intricacy of manufacturing activities and dimension of lean implementation.

vii) Finding suggested lean strategies using model: The DST created is used for calculation of the augmented perceived value of diminishing distinguished manufacturing wastes by choosing the proper lean strategies. A MATLAB program was created under DST to locate the optimum number of lean strategies for the distinguished wastes. Producer's distributed cost and time for enhancing their wasteful aspects are utilized as cost and time limitations

Table 2: Solution with given Set of Constraints

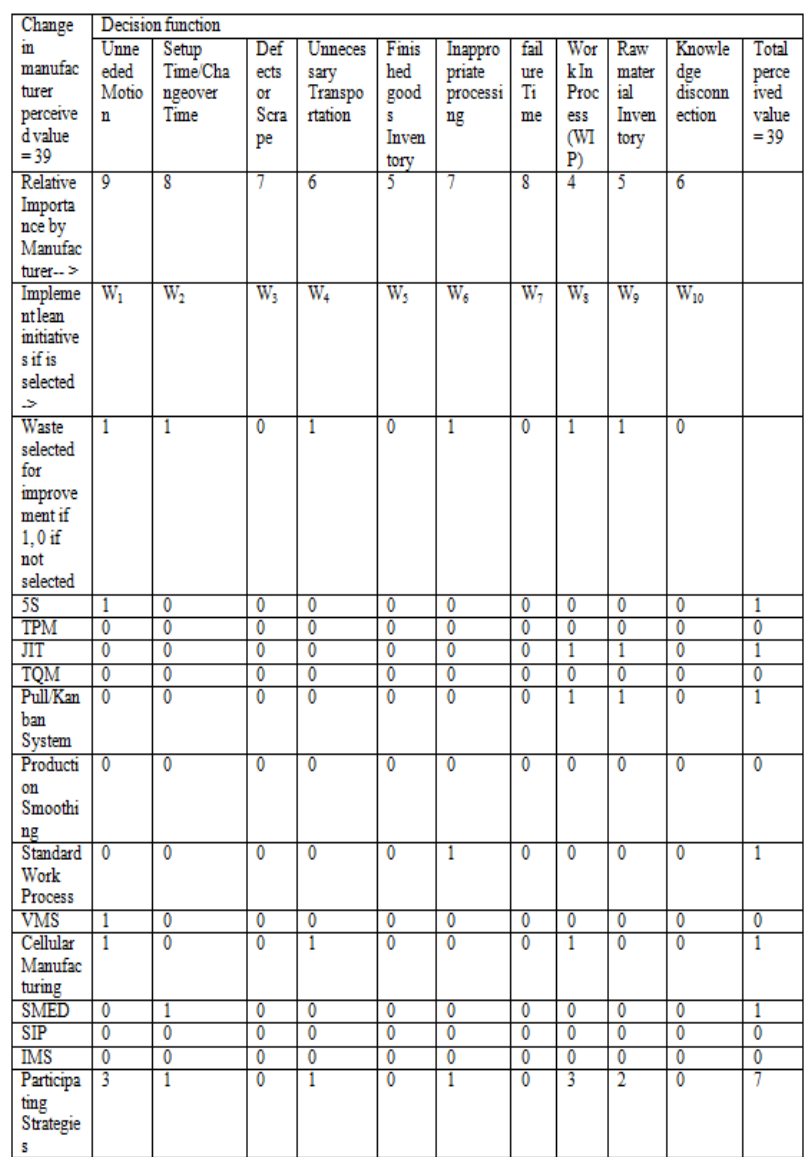

Unseemly processing can be decreased by applying Standard Work Process. Improper processing is firmly identified with working system of operators or awful structure of the product. Therefore, strategy time estimation (MTM) could be a decent answer for this waste. At last, SMED implementation diminishes the setup time wastes. Therefore, the request of lean strategies ought to be Cellular Manufacturing, JIT or Kanban, 5S or Visual Management system followed by SMED. The diverse cost and time units which are utilized as limitations are given by the producers. Along these lines, the genuine measure of cost and time required to execute these chose lean strategies is controlled by applying the philosophy and MATLAB program.

The slack value between the real implementation cost and time and given imperatives can be determined by substituting required units (Column 3) for accessible units (Column 2). Table 2 demonstrates the graphical portrayal of various lean strategies and wastes and perceived viability values as indicated by $\mathrm{ABC}$ 's resource limitations .

\section{CONCLUSION}

This approach can be utilized to settle $\mathrm{f}$ organization isn't just to amplify the apparent esteem yet additionally to drive the choice dependent on policies. This examination propels the present information base by creating mathematical models and methodologies to conquer lean strategy selection and leanness appraisal issues. By choosing suitable lean strategies, a manufacturer can all the more likely organize implementation endeavors and assets to expand the advantages of actualizing lean strategies in their organization. The leanness record is utilized to assess an organization's current (before lean implementation) leanness state against the state after lean implementation and to build up benchmarking (the ideal leanness state). Henceforth, this examination gives a persistent enhancement apparatus for a lean manufacturing organization.

\section{REFERENCES}

1. Pentlicki, J.H. (2014). Barriers and Success Strategies for Sustainable Lean Manufacturing Implementation: A Qualitative Case Study, PhD thesis, University of Phoenix, Phoenix

2. Bortolotti, T., Boscari, S., Danese, P. (2015). Successful lean implementation: organizational culture and soft lean practices. International Journal of Production Economics, vol. 160, p. 182-201, DOI:10.1016/j.ijpe.2014.10.013

3. Kull, T.J., Yan, T., Liu, Z., Wacker, J.G. (2016). The moderation of lean manufacturing effectiveness by dimensions of national culture: Testing practice-culture congruence hypotheses. International Journal of Production Economics, vol. 153, p. 1-12, DOI:10.1080/00207543.2014.903348

4. Jekiel, C.M. (2017). Lean Human Resources: Redesigning HR Processes for a Culture of Continuous Improvement. Productivity Press, New York.

5. Liker, J.K. (2014). The Toyota way. New Delhi, India: Tata McGraw-Hill.

6. Pieterse, K. (2015). Leaning the South African way: Implementing lean manufacturing in the rainbow country. Port Elizabeth, South Africa: Trilean Publishing

7. Hunter, Steve L., Forest products Journal, 54(4), 2014, 32-39.

8. Koragaokar. M.G., Just in Time Manufacturing, Macmillan publications, Reprinted, 2015

9. Lin, WD, Jin, X \& Chia, SY 2014, 'Simulation Based Lean Six Sigma Approach to Reduce Patients Waiting Time in an Outpatient Eye Clinic', Proceedings of the 2014 IEEE, pp. 394-398.

10. Gautam, N., \& Singh, N. (2008). Lean product development: Maximizing the customer perceived value through design change (redesign). International Journal of Production Economics, 114(1), 313-332 